The Blood Research Foundation Center, ${ }^{1}$ Ospedale di Tortona (Alessandria)

\title{
Introduction to the General Discussion on the Gytological and Haematological Effects of Antimitotics
}

\section{G. Astaldi}

I will just make a few remarks before opening this General Discussion on the Cytological and Haematological effects of Antimitotics. I believe that numerous and interesting will be your comments. Let me first go back very briefly to the pioneer of these compounds, colchicine, already mentioned in the Egyptian, Greek, and Roman Pharmacology, but whose stathmokinetic effect was illustrated by P. Dustin Sr. just in 1934. We are lucky enough to have in our program the Dustin name perpetrated through his son, A. P. Dustin Jr.

Several years ago, since 1947 on, my group adopted colchicine to perform a test for evaluating in tissue culture the proliferative activity of the blood-forming cells from different physio-pathologic conditions. Using that method, among other it was possible to ascertain that - contrary to expectations - the proliferative activity of the bone marrow cells in human leukaemias is rather low, and this finding has been later confirmed by many investigators using different approachments and methods. Quite recently, in collaboration with Gociu M. and Airò R., I have started investigating the effect of colchicine on the blastic transformation of human peripheral blood lymphocytes stimulated with Phytohaemagglutinin (Phyto) in cell culture. The main purposes of this research project are:

I. To test the effect of colchicine on the blastic development process, which occurs as a consequence of such an intense RNA- and DNA- synthesis presented by human lymphocytes in the Phyto-culture system;

2. To evaluate the extent of proliferation of the Phyto-stimulated lymphocytes, because the divisions which occur in these cells could definitely contribute in making higher their number. (In fact, the number of the blast-like cells obtained after 3-4 days of cell culture with Phyto is a function not only of the number of the lymphocytes which undergo blastic transformation, but also of the number of the same blastlike cells which undergo mitotic division).

In these experiments, colchicine has been added to the Phyto-culture system at the early beginning of the culture, and the drug concentration was 0.25 gammas $/ \mathrm{ml}$.

1 Supported by «The Blood Research Foundation», Washington, D.G., USA 
On the other hand, colchicine was not added to the control culture. Periodically every $12 \mathrm{hrs}$, until $96 \mathrm{hrs,} \mathrm{samples} \mathrm{of} \mathrm{cells} \mathrm{were} \mathrm{taken} \mathrm{from} \mathrm{these} \mathrm{cultures,} \mathrm{and} \mathrm{smeared}$ and stained with M.G.G. Both the percentages of blast-like cells among all the lymphatic cell population, and the percentages of mitoses among the same blasts were determined on these smears.

From the results, taken as a whole, it appeared that no significant difference does exist between the extent of blasts developing in the control-cultures on one hand, and the extent of blasts developing in the colchicine-cultures on the other, during the first $24-36 \mathrm{hrs}$ of cultivation, i. e. during the period of the RNA-synthesis in the Phyto-stimulated lymphocytes. On the contrary, from $48 \mathrm{hrs}$ on, a significant difference is evident, as a consequence of the fact that in the control-cultures the blast-like cells underwent divisions, whereas in the colchicine-cultures blast divisions did not occur.

These preliminary results seem to show that: I) Colchicine (at the experimented dose, which was sufficient to block in premetaphase all the blasts entering mitosis) does not interfere with the capacity of normal human lymphocytes to undergo the blastic transformation in the Phyto-culture system. 2) The experimented method may provide some information for explaining the number of the blast-like cells obtained in a 3-days culture. In details, this method may help in judging the extent of blasts derived through the transformation process of lymphocytes and, on the other hand, derived through mitotic divisions. 